\title{
Tecnura
}

\section{Interfaces adaptativas personalizadas para brindar recomendaciones en repositorios de objetos de aprendizaje}

\section{Personalized adlaptive interfaces for supporting recommendation from learning object repositories}

\author{
Oscar Mauricio Salazar Ospina, ${ }^{1}$ Paula Andrea Rodríguez Marín, ${ }^{2}$ \\ Demetrio Arturo Ovalle Carranza, ${ }^{3}$ Néstor Darío Duque Méndez ${ }^{4}$
}

Fecha de recepción: 29 de septiembre de 2015

Fecha de aceptación: 18 de noviembre de 2017

Cómo citar: Salazar, O., Rodríguez, P., Ovalle, D. y Duque, N. (2017). Interfaces adaptativas personalizadas para brindar recomendaciones en repositorios de objetos de aprendizaje. Revista Tecnura, 21(53), 107-118, doi: $10.14483 / 22487638.9287$

\section{Resumen}

Contexto: existen muchos repositorios de recursos educativos que permiten buscar y recuperar objetos de aprendizaje, de esta forma se puede tener acceso a millones de recursos educativos; sin embargo, se requiere mejorar la presentación, visualización y satisfacción de uso de dichos objetos de aprendizaje, teniendo en cuenta las preferencias y necesidades de los estudiantes.

Método: el objetivo de este artículo es incorporar una interfaz adaptativa personalizada a un sistema multiagente con el fin de recomendar objetos de aprendizaje, desde repositorios locales y remotos utilizando el perfil cognitivo de los estudiantes.

Resultados: la validación del prototipo se realizó a través de un caso de estudio en el cual la interfaz adaptó tanto la presentación como la visualización de los objetos de aprendizaje a través de las preferencias, necesidades y características de los estudiantes.
Conclusiones: se puede concluir que las interfaces adaptativas personalizadas demuestran su eficacia y representan entonces un gran aporte en los entornos de e-learning, debido a que modifican en tiempo real la visualización y la presentación, teniendo en cuenta el perfil cognitivo del aprendiz.

Palabras clave: estilos de aprendizaje, interfaces adaptativas personalizadas, perfiles de estudiante, repositorios de objetos de aprendizaje, sistemas de recomendación, sistemas multiagente.

\section{Abstract}

Context: There are many repositories that allow searching and retrieving learning objects, so a lot of learning resources can be accessed. However, it is required to improve the presentation and visualization of those learning resources considering the student's preferences, needs, and cognitive features.

Method: The aim of this paper is to incorporate a customized interface with an adaptive multi-agent

1 Ingeniero de Sistemas e Informática, magíster y candidato a doctor en Ingeniería de Sistemas e Informática. Universidad Nacional de Colombia. Medellín, Colombia. Contacto: omsalazaro@unal.edu.co

2 Administradora de Sistemas Informáticos, magíster y candidata a doctora en Ingeniería de Sistemas e Informática. Universidad Nacional de Colombia. Medellín, Colombia. Contacto: parodriguezma@unal.edu.co

3 Ingeniero de Sistemas y Computación, magíster (DEA) y doctor en Informatique. Profesor titular del departamento de Ciencias de la Computación y de la Decisión Universidad Nacional de Colombia. Medellín, Colombia. Contacto: dovalle@unal.edu.co

4 Ingeniero mecánico, magíster y doctor en Ingeniería de Sistemas e Informática. Profesor Asociado del departamento de Informática y Computación Universidad Nacional de Colombia. Manizales, Colombia. Contacto: ndduqueme@unal.edu.co 
system for learning objects recommendation from local and remote repositories based on the student's cognitive profile.

Results: The prototype validation was made through a case study in which the interface has adapted not only the presentation but the visualization of learning objects taking into account the student's preferences, needs and cognitive features.

Conclusions: We can conclude that personalized adaptive interfaces demonstrate their efficacy and represent a great contribution to e-learning environments since they modify in real time the visualization and presentation of educational resources using the student's cognitive profile.

Keywords: Learning styles, multi-agent systems, personalized adaptive interfaces, recommendation systems, repositories of learning objects, student profiles.

\section{INTRODUCCIÓN}

Las interfaces de usuario han evolucionado en las últimas décadas, desde interfaces eminentemente textuales a interfaces más complejas de tipo multimodal (integrando diversas formas de interacción tales como multimedia, táctil, vocal, etc.); lo anterior ha permitido mejorar la interacción hombre-máquina. Según López et al., en se debe distinguir entre adaptabilidad y adaptatividad en las interfaces de usuario: en una interfaz adaptable el usuario es quien explícitamente adapta la interfaz para que se ajuste a sus gustos y características, por ejemplo, los gestores de ventanas pueden permitir al usuario cambiar la configuración del aspecto del escritorio con respecto a colores, fuentes, fondo del escritorio o comportamiento de algunos de sus componentes; en contraste, en una interfaz adaptativa, el mismo sistema es el actor responsable de activar las acciones necesarias para realizar la adaptación, así, por ejemplo, cuando en un procesador de texto se detecta un error gramatical el mismo procesador automáticamente lo marca o incluso lo corrige sin intervención humana.

Las interfaces adaptativas personalizadas se pueden definir entonces como aquellos parámetros de la interfaz que se adecuan automáticamente a las características de los usuarios (Bolotnikova, Gavrilova, y Gorovoy, 2011), permitiendo la mejora en la satisfacción y la permanencia del usuario interactuando con la aplicación o en el sitio web.
Las tendencias actuales van hacia los sistemas de recuperación de información web que permitan adaptar los resultados mediante interfaces adaptativas personalizadas, los cuales tienen en cuenta las propiedades y contextos de los usuarios (Smith, McGinty, Reilly, y McCarthy, 2004; Steichen, Ashman, y Wade, 2012).

Por su parte, los objetos de aprendizaje (OA) son definidos por la IEEE como entidades digitales que cuentan con propiedades de diseño instruccional, los cuales pueden utilizarse, reutilizarse o referenciarse durante un procese de aprendizaje soportado por computador (IEEE, 2002) y se diferencian de otros recursos educativos tradicionales ya que están disponibles inmediatamente en la web. Los OA cuentan con metadatos descriptivos y están almacenados en repositorios donde se pueden realizar búsquedas por medio de estos; además, existen las federaciones de repositorios con el objetivo de maximizar la cantidad de OA a los que un estudiante puede tener acceso, permite también tener acceso a los recursos de otros repositorios y compartir recursos a través de un punto único de acceso (Van de Sompel, Chute, y Hochstenbach, 2008).

Tener un perfil de usuario permite identificar las necesidades, preferencias y características del estudiante que está haciendo una búsqueda de OA, ayudándolo para obtener resultados de búsqueda cercanos a sus características y adaptar la visualización de la interfaz; por otro lado, conocer al 
usuario a través de un perfil favorece a los estudiantes y profesores en la elección de OA acordes a su especificidad (Rosado y Verjel, 2015; Kritikou, y otros, 2008). Así entonces, utilizando sistemas de recomendación basados en el perfil de los usuarios, permite que ellos tengan una visión personalizada sobre grandes conjuntos de productos (Becerra y otros, 2015) en este caso de OA; adicionalmente, con las características del perfil se puede hacer una personalización del repositorio y hacer más agradable su visita al sitio. Los agentes inteligentes que provienen de la inteligencia artificial distribuida son estructuras computacionales caracterizadas por exhibir proactividad, autonomía y flexibilidad con el fin de realizar tareas específicas que requieren un mínimo de intervención humana (Wooldridge, Jennings, y Kinny, 1999). Estos agentes se están empleando casi de igual manera que los sistemas tradicionales, convirtiéndose en una muy buena opción para resolver problemas donde se necesita de sistemas autónomos que trabajen individualmente y se cooperen entre ellos para lograr una meta común; adicionalmente, modelan el problema a través de distribución de tareas y conocimientos, lo cual es de gran utilidad cuando se plantea el enfoque de solución con dispositivos móviles y computación ubicua.

"La web semántica es una extensión de la web actual en donde la información tiene un significado bien definido, es más entendible por los computadores y en donde las personas pueden trabajar cooperativa y colaborativamente" (Berners-Lee y Hendler, 2001); las ontologías se convierten así en la forma de representar semánticamente el conocimiento disponible en internet, lo cual permite la reutilización y accesibilidad a partir de buscadores y programas computacionales de recuperación de información.

“Una ontología es el resultado de seleccionar un dominio y aplicar sobre el mismo un método para obtener una representación formal de los conceptos que contiene y las relaciones que existen entre estos" (Liang, Zhu, Tian, y Ji, 2010); cabe señalar que las ontologías se especifican a través de lenguajes computacionales tales como OWL (por sus siglas en inglés para Web Ontology Language), con el fin de automatizar el procesamiento de la información por parte de programas basados en la web, en vez de que sea realizado en forma manual como era el caso en la web tradicional.

A continuación, se presentan algunos trabajos relacionados con el desarrollo de interfaces adaptativas personalizadas para brindar recomendaciones.

Papanikolaou et al. (2002), describen el prototipo INSPIRE con el fin de personalizar la interacción en un sistema hipermedia educativo que se basa en la web, enfatizan que los estudiantes integran las ideas desde las teorías a través de su propio estilo, su objetivo principal es entregar una secuencia de tareas que coincidan con la manera de estudiar de cada alumno; del estudiante tienen en cuenta los conocimientos previos de los conceptos del dominio y el estilo de aprendizaje, evaluando las actitudes de los estudiantes hacia el diseño instruccional que se le propone, en el modelo de aprendizaje se almacena la información acerca de la interacción del alumno con el contenido. Aunque es un sistema que realiza recomendaciones de objetos de aprendizaje, basado en objetivos de aprendizaje que maneja los estilos de aprendizaje, donde se tiene en cuenta las interacciones del estudiante, no se realizan adaptaciones de la interfaz (Papanikolaou, Grigoriadou, Kornilakis, y Magoulas, 2002).

Uruchrutu, MacKinnon, y Rist (2005), realizan un estudio para evaluar el diseño de interfaz según el estilo cognitivo de aprendizaje de un estudiante para facilitar el rendimiento en su aprendizaje, concluyen que se deben considerar las diferencias individuales para elaborar modelos de usuario y así proporcionar entornos de aprendizaje adaptados; además, con el análisis de los datos se discute que el estilo de la interfaz tiene un impacto en las preferencias de los estudiantes, que a su vez tienen un impacto en su capacidad de aprendizaje, sin embargo, no trabajan con repositorios de objetos de aprendizaje. 
Peissner y Edlin-White (2013), proponen interfaces adaptativas para usuarios con necesidades especiales, presentan los requisitos esenciales de los sistemas adaptativos, usando los patrones de diseño de interacción para la implementar interfaces de usuario, con el fin de mejorar la accesibilidad a un máximo de la heterogeneidad de los grupos de usuarios. Aunque es un estudio preliminar para el desarrollo modular de interfaces adaptativas según características de usuarios (necesidades especiales), no trabajan sobre materiales de educación almacenados en repositorios de objetos de aprendizaje y no utilizan el estilo de aprendizaje como el componente con más relevancia.

Kolas y Staupe (2007) presentan una interfaz personalizada para apoyar el proceso de e-learning, esta tiene la capacidad de adaptar OA y actividades de aprendizaje de acuerdo a las características de los estudiantes, considerando también las áreas de interés y el nivel de experticia en dichas áreas. Es importante denotar que esta investigación tiene en cuenta varias características del perfil, sin embargo, no considera gustos visuales de los estudiantes tales como color y tamaño de las fuentes, tipos de recursos, formatos, etc.

Una arquitectura de alto nivel para entregar interfaces adaptativas en dispositivos móviles es presentada en Nivethika, Vithiya, Anntharshika, y Deegalla (2013), este trabajo tiene en cuenta interacciones pasadas del usuario con el dispositivo para poder adaptar de mejor manera las interfaces, de esta manera, el Framework incluye una serie de pasos de preprocesamiento de la información, de aprendizaje y de renderización de la interfaz; también considera el nivel de experticia del usuario dentro de una aplicación, así es capaz de determinar si es un usuario nuevo para ofrecer mensajes de ayuda que le permitan navegar en la aplicación. Como falencia, esta arquitectura no considera recomendación de recursos educativos o de otra naturaleza y tampoco establece un esquema de representación del conocimiento referente a gustos y limitaciones de los usuarios.
Lee, Choi, y Kim (2011) desarrollan un prototipo de interfaz para dispositivos móviles con sistema operativo Android, el cual permite recomendar aplicaciones teniendo en cuenta el contexto tanto espacial como temporal de los usuarios; este trabajo de investigación utiliza criterios tales como clima, ubicación, tiempo, entre otros, para recomendar las aplicaciones que más se ajusten al perfil del usuario en tiempo real. Es así como la aplicación busca saber lo que el usuario quiere, ahorrándole tiempo en la búsqueda de las aplicaciones; sin embargo, este trabajo a pesar de que tiene en cuenta los perfiles de los usuarios debería considerar aspectos como los recursos educativos que serían útiles recomendar.

El objetivo de este artículo es entonces incorporar una interfaz adaptativa personalizada a un sistema multiagente que recomiende $\mathrm{OA}$ a estudiantes (Rodriguez, Tabares, Duque, Ovalle, y Vicari, 2013), almacenados en repositorios heterogéneos incluyendo federaciones de repositorios, teniendo en cuenta las preferencias, características y necesidades de los estudiantes; lo anterior con el fin de mejorar la presentación, visualización y satisfacción de uso de dichos contenidos educativos. La validación del prototipo se realizó a través de un caso de estudio que demuestra la eficacia de la utilización de interfaces adaptativas personalizadas basadas en agentes en entornos de aprendizaje virtual.

Este artículo se organiza así: en la segunda sección se expone el marco conceptual de la investigación, en la tercera se presentan los trabajos relacionados, en la cuarta se plantea el diseño del SMA propuesto con interfaz adaptativa, posteriormente, en la quinta sección se presenta un caso de estudio y, por último, se presentan las conclusiones y el trabajo futuro propuesto.

\section{METODOLOGÍA}

Esta sección presenta el proceso la incorporación de una interfaz adaptativa y personalizada a un sistema multiagente que realiza recomendaciones 
de objetos de aprendizaje, desde repositorios locales y remotos; dicha interfaz considera las preferencias y necesidades de los estudiantes con el fin de mejorar la presentación, visualización y satisfacción de uso de dichos contenidos educativos. El desarrollo, implementación e integración del sistema consideró una metodología iterativa que comprendió las siguientes fases: (a) plantear e implementar una ontología que permita representar el conocimiento asociado a perfiles de usuario al igual que los metadatos que describen los OA; (b) analizar y diseñar un SMA de recomendación de OA a partir del modelo ontológico propuesto; (c) diseñar e implementar una interfaz adaptativa que se acople al modelo multiagente y que permita personalizar sus componentes principales basado en las características descritas en los perfiles de usuario. A continuación, se describe el proceso de desarrollo que enmarca cada uno de los componentes del sistema.

\section{Modelo ontológico del perfil del aprendiz}

Se hace indispensable contar con un mecanismo de representación del conocimiento para inicialmente adaptar las interfaces gráficas y, posteriormente, realizar las recomendaciones de recursos educativos, en el cual se definen las características del estudiante, además de sus gustos y limitaciones; con base en esto, se decidió definir una ontología que permitiera no solo representar dicho conocimiento sino también realizar inferencias a partir de una estructura estandarizada, portable y extensible. La figura 1 presenta la estructura ontológica definida para representar el conocimiento del SMA.

En primera instancia se define la estructura de los perfiles de los estudiantes, donde se tienen almacenados datos personales como su nombre y fecha de nacimiento, aspectos académicos ligados a su historia académica, datos relacionados con

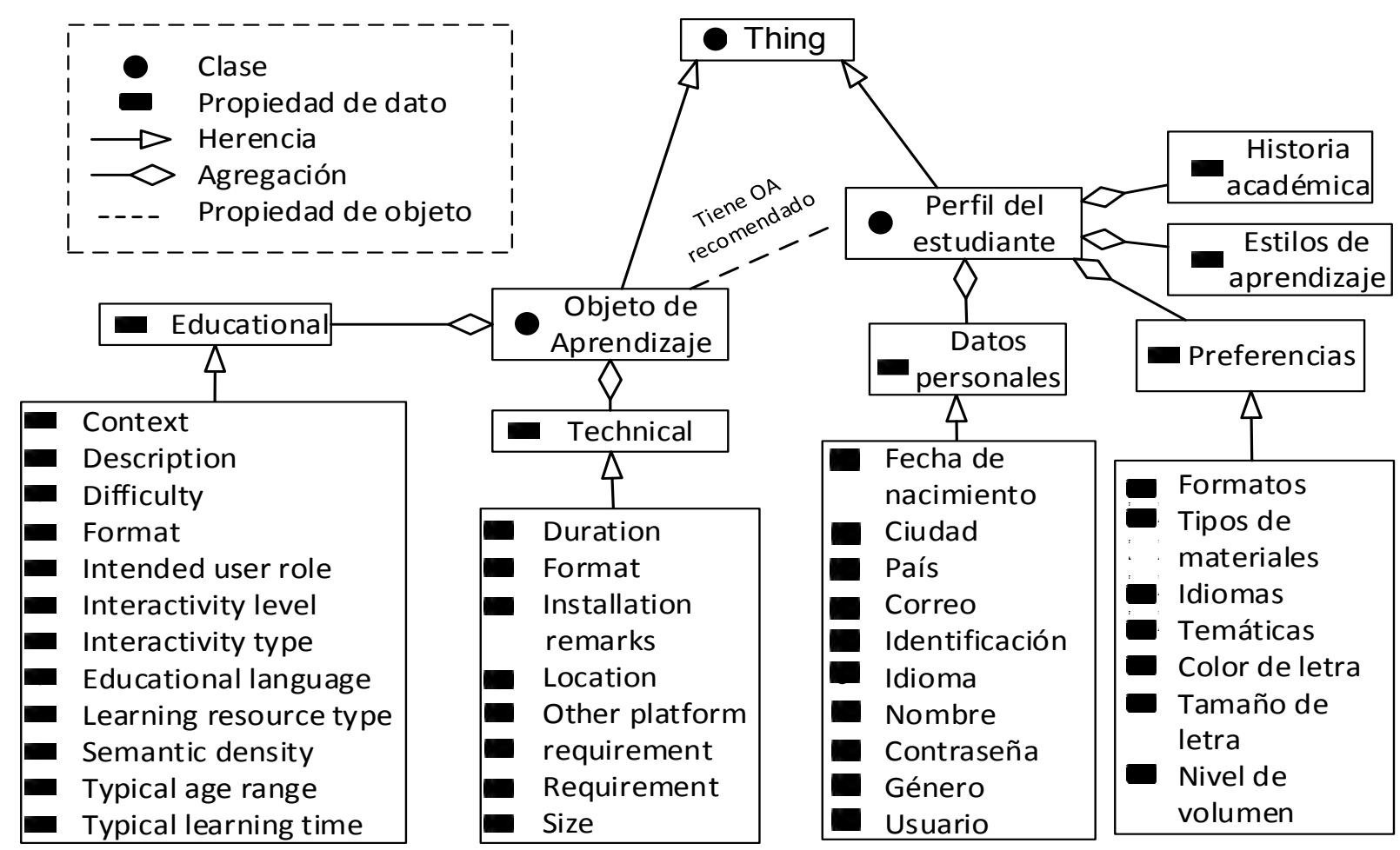

Figura 1. Modelo Ontológico del SMA

Fuente: elaboración propia 
preferencias visuales y de contenido, y aspectos psicopedagógicos como el estilo de aprendizaje. Las demás entidades de la ontología están relacionadas con los componentes technical y educational del estándar IEEE-LOM, utilizados para adaptar los OA al perfil del estudiante.

\section{Arquitectura BROA con interfaz adaptativa}

BROA es un sistema multiagente que permite la búsqueda, la recuperación, la recomendación y la evaluación de OA, el sistema inicia por una cadena de búsqueda que ingresa el usuario y son recuperados los OA; cabe señalar que estos objetos pasan por un filtrado adicional que es la recomendación, la cual consiste en buscar aquellos que cumplan con el estilo de aprendizaje del estudiante y aqueIlos que fueron evaluados por estudiantes similares.

Se realiza la búsqueda en repositorios heterogéneos o en federaciones de repositorios a través de los metadatos que describen cada objeto, y, debido a que los ROA están distribuidos, son diferentes en su diseño, estructura, y no manejan los mismos estándares de metadatos, se tiene por cada uno un agente que conozca cómo están almacenados los OA y cómo es su forma de acceso y recuperación, con el fin de facilitar las búsquedas. El sistema se construyó como un sistema multiagente porque este enfoque permite realizar en paralelo la búsqueda en diferentes ROA, además estos sistemas pueden realizar deliberación para tomar la decisión sobre en qué ROA se debe realizar la búsqueda y qué recomendaciones realizar al estudiante, por otra parte, se pueden identificar los problemas a resolver por cada agente, definir sus límites y especificar la cooperación, la coordinación y la distribución de tareas. En la figura 2, se exhibe la arquitectura del SMA propuesto y cada uno de sus componentes.

La arquitectura del sistema se describe a continuación a partir de cada uno de los agentes que la componen y sus interacciones.

Agente interfaz: tiene como rol principal realizar la adaptación de la interfaz del repositorio, según las características que definen el perfil del estudiante que está realizando las búsquedas; se comunica con el agente usuario para conocer las preferencias del usuario tales como formatos de los archivos, idiomas, tipos de materiales, color y tamaño de letra y nivel del volumen para los contenidos auditivos. De igual manera, se comunica con el agente

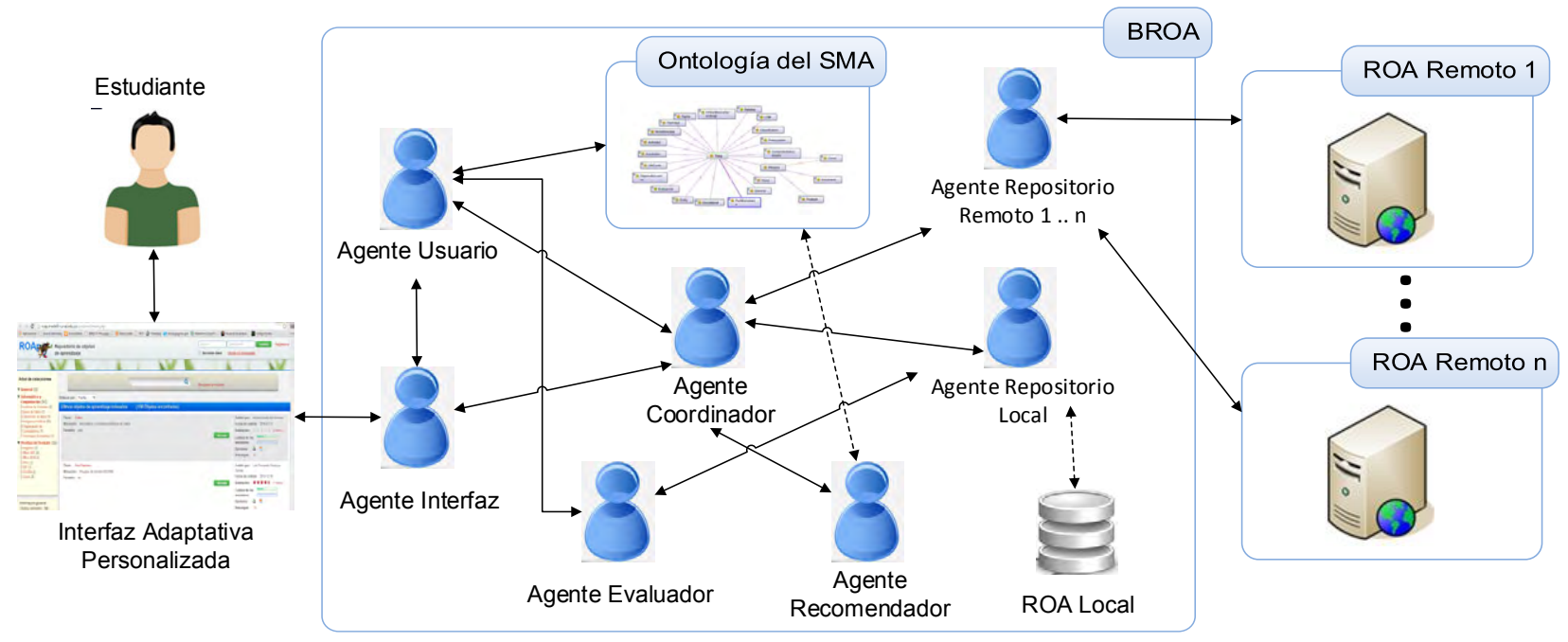

Figura 2. Arquitectura del SMA

Fuente: elaboración propia 
coordinador, quien es responsable de entregar los OA resultantes después de realizada la búsqueda.

Agente usuario: su función principal es la de representar al usuario humano dentro del SMA, es decir, gestiona el perfil del usuario, lo que implica crear y modificar tanto las características como sus preferencias; luego, envía la cadena de consulta para que el agente coordinador realice la búsqueda y permite evaluar los OA que son recomendados. De igual manera, este agente realiza inferencias a la ontología utilizando el lenguaje de consultas ontológicas SPARQL, con el fin de enviar características importantes al agente interfaz y este a su vez adapte los contenidos visuales.

Agente coordinador: este agente es deliberativo ya que se encarga de redireccionar las consultas que hace el usuario al repositorio local y a los remotos para realizar la búsqueda; este agente conoce los repositorios asociados al sistema y cuales manejan que información. Además, accede a la información del agente usuario para conocer las características útiles para la recomendación (estilo de aprendizaje, grado de escolaridad, preferencia de idioma). Los resultados los entrega al agente interfaz.

Agente repositorio local y agentes repositorios remotos: son los encargados de hacer las búsquedas propiamente en los repositorios, tanto de forma local como remota; estos agentes conocen cómo están almacenados los $\mathrm{OA}$ en el repositorio respectivo, bajo qué estándar y el tipo de metadatos que maneja, también conoce el tipo de búsqueda que se puede realizar dentro del repositorio y cómo recuperar los OA que hay en él. Cabe señalar que el agente del repositorio local se encarga además de almacenar la evaluación que le entrega el agente evaluador de cada $\mathrm{OA}$; igualmente, en la arquitectura propuesta existe un agente por cada repositorio local o remoto.

Agente de recomendación: este agente realiza el proceso de recomendación sobre los metadatos que describen los OA y en las informaciones de estilo de aprendizaje, grado de escolaridad y preferencia de idioma del estudiante registrado. Se buscan aquellos OA acordes al perfil del estudiante.

Agente evaluador: es el agente que se encarga de gestionar las evaluaciones que realiza un usuario a algún OA que ha sido explorado; se comunica así con el agente ROA local para almacenar estas evaluaciones, producto de la calificación explicita que da el estudiante seleccionando una puntuación de uno a cinco de acuerdo a su nivel de satisfacción respecto a ese $\mathrm{OA}$, donde cinco es muy satisfecho y uno muy insatisfecho.

La figura 3 presenta el mecanismo de comunicación entre cada uno de los agentes descritos

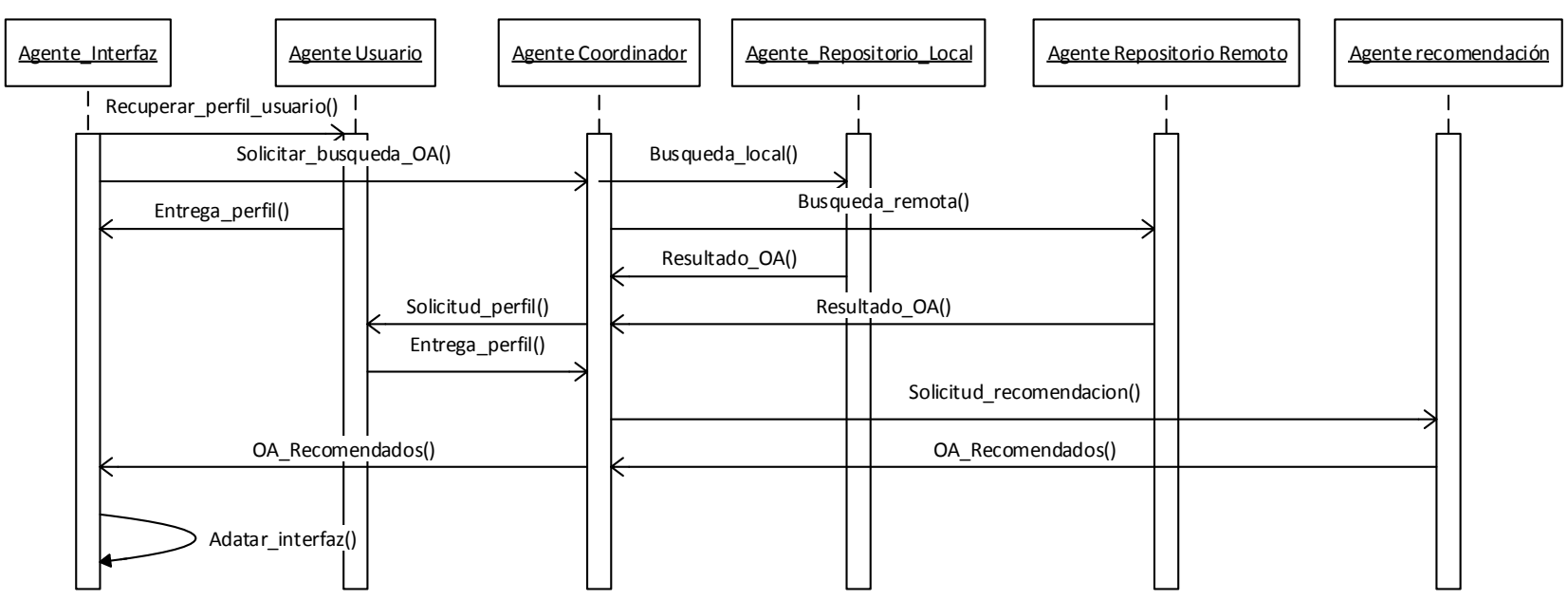

Figura 3. Diagrama de secuencias de la comunicación entre agentes para la adaptación de la interfaz gráfica del repositorio

Fuente: elaboración propia 
previamente para realizar la adaptación de la interfaz de usuario del repositorio de OA; es importante resaltar que, aunque se evidencia un proceso secuencial, este solo representa una interacción entre el estudiante y el sistema, es decir, la fortaleza del sistema multiagente está en la distribución de tareas con el fin de atender diversas solicitudes en un mismo intervalo de tiempo sin que el usuario se vea afectado.

\section{RESULTADOS}

A continuación, se explican los principales Framework utilizados para la implementación del prototipo de validación.

Java Agent Development Framework (JADE, por sus siglas en inglés) es una plataforma que pone a disposición diversos recursos computacionales que facilitan la construcción de entornos computacionales basados en agentes, los cuales pueden ser utilizados en aplicaciones de e-learning (Bellifemine, Poggi, y Rimassa, 1999); a partir de esto, se utilizó esta tecnología para implementar la plataforma multiagente, debido a que está desarroIlada en Java, lo que permite la fácil integración con otras herramientas y tecnologías. Para el desarrollo e implementación de la ontología se utilizó Protegé, una herramienta de código abierto desarrollada en Java, la cual permite el desarrollo de ontologías OWL. Posteriormente, y luego de tener el archivo OWL con la estructura ontológica, se procedió a integrarlo con el SMA a través del Framework JENA, mediante el cual se crearon las

Tabla 1. Tabla de valores para el caso de estudio realizado

\begin{tabular}{ll}
\hline & Caso de estudio \\
\hline Formatos & jpg - pdf-png \\
\hline Idiomas & Inglés \\
\hline Tamaño de letra & Grande \\
\hline Color de letra & Oscura \\
\hline Tipos de materiales & Visuales-Textuales \\
\hline Nivel de volumen & Alto \\
\hline
\end{tabular}

Fuente: elaboración propia instancias y se realizaron las inferencias utilizando consultas SPARQL. Finalmente, el repositorio local está implementada con el gestor de bases de datos PostgreSQL que se caracteriza por ser un sistema estable, de alto rendimiento y gran flexibilidad.

Para el caso de estudio aplicado al prototipo implementado se seleccionó un perfil de estudiante aleatorio, el cual permitió evaluar qué tan bien se adapta la interfaz a las necesidades y los requerimientos del estudiante. La tabla 1 presenta los valores de preferencias y gustos de dicho perfil, los cuales serán tenidos en cuenta para adaptar tanto los OA presentados en la interfaz principal del SMA, como los aspectos visuales de esta interfaz. BROA entrega a través del agente de interfaz un listado de objetos recomendados al usuario, que son aquellos OA que se adaptan a su estilo de aprendizaje, características, necesidades y preferencias.

Con base en el perfil seleccionado, el sistema adaptó adecuadamente los componentes en la interfaz principal. La figura 4 presenta la interfaz original del sistema, la cual se transforma en la nueva interfaz adaptativa del sistema BROA que se presenta en la figura 5, esta incluye modificaciones respecto a idioma, color y tamaño del texto, recomendaciones iniciales de OA que pueden ser de interés al estudiante de acuerdo a su perfil y materiales adaptados a sus preferencias. Las acciones anteriores son realizadas por el agente de interfaz en coordinación estrecha con el agente recomendador; de igual manera, el SMA a través del agente interfaz, es capaz de adaptar los contenidos en el momento que el estudiante decide accederlos, es decir, cuando el usuario decide acceder a un OA, el SMA está en la capacidad de activar automáticamente características del contenido, por ejemplo, si el estudiante accede un OA visual-auditivo, el SMA activa los subtítulos o acomoda el tamaño de la pantalla si el estudiante así lo desea.

A partir del caso de estudio realizado y del perfil seleccionado para este, los resultados fueron divididos en dos frentes: el primero de ellos fue la adaptación de la interfaz del sistema BROA, personalizando aspectos como color y tamaño del 
texto, idioma, nivel de volumen auditivo y distribución del contenido; mientras que, en el segundo, se realizó una recomendación de OA inicial, teniendo en cuenta calificaciones previas, tipos de materiales y formatos preferidos. Es importante resaltar que algunas de las características tomadas del perfil del estudiante ya habían sido establecidas previamente, por ejemplo, el estilo de aprendizaje fue identificado mediante un test realizado al estudiante al momento del registro.

Adicionalmente, cabe señalar se tiene un agente por cada repositorio asociado al sistema, que conoce cómo están almacenados los OA y cómo es su forma de acceso y recuperación, con el fin de facilitar las búsquedas; las búsquedas se realizan en un ROA local, donde están los OA que ya fueron evaluados y en los ROA remotos asociados al sistema.
El agente interfaz es clave para el desempeño de la interfaz adaptativa, pues realiza adaptaciones visuales y de contenido; las visuales se refieren a características gráficas de la interfaz principal como texto e imágenes, mientras que las de contenido permiten ofrecer OA de interés para el usuario sin que este lo solicite, exhibiendo así características de proactividad, por ejemplo, si el usuario desea acceder OA visuales y/o auditivos, el agente permite adaptar el nivel de volumen sonoro, los subtítulos del contenido del OA y el tamaño de la pantalla. La Figura 5 exhibe la nueva interfaz adaptada obtenida del sistema BROA considerando los aspectos cognitivos del estudiante (e.g. estilo de aprendizaje, preferencias, gustos cromáticos, limitaciones auditivas, etc) la cual se transformó a partir de la interfaz original presentada en la figura 4.

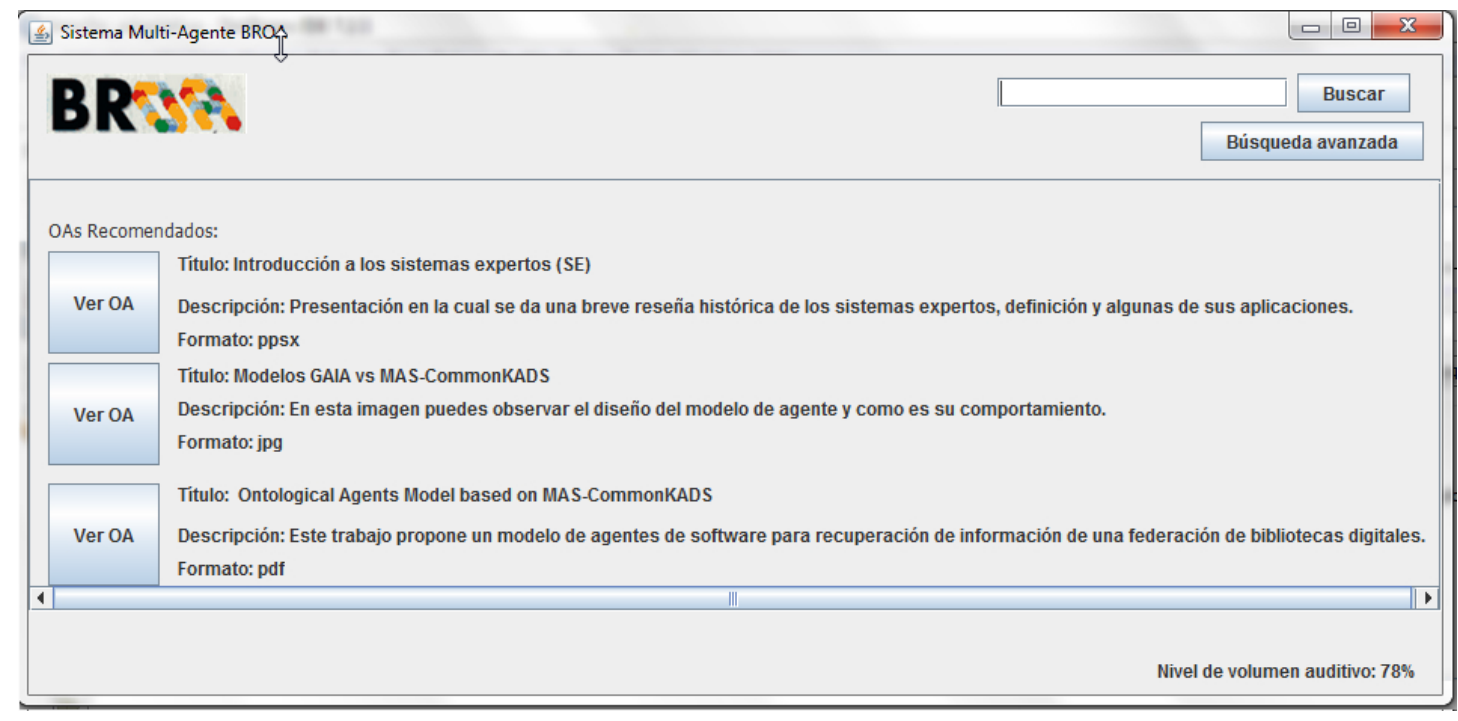

Figura 4. Interfaz original sin adaptar

Fuente: elaboración propia 


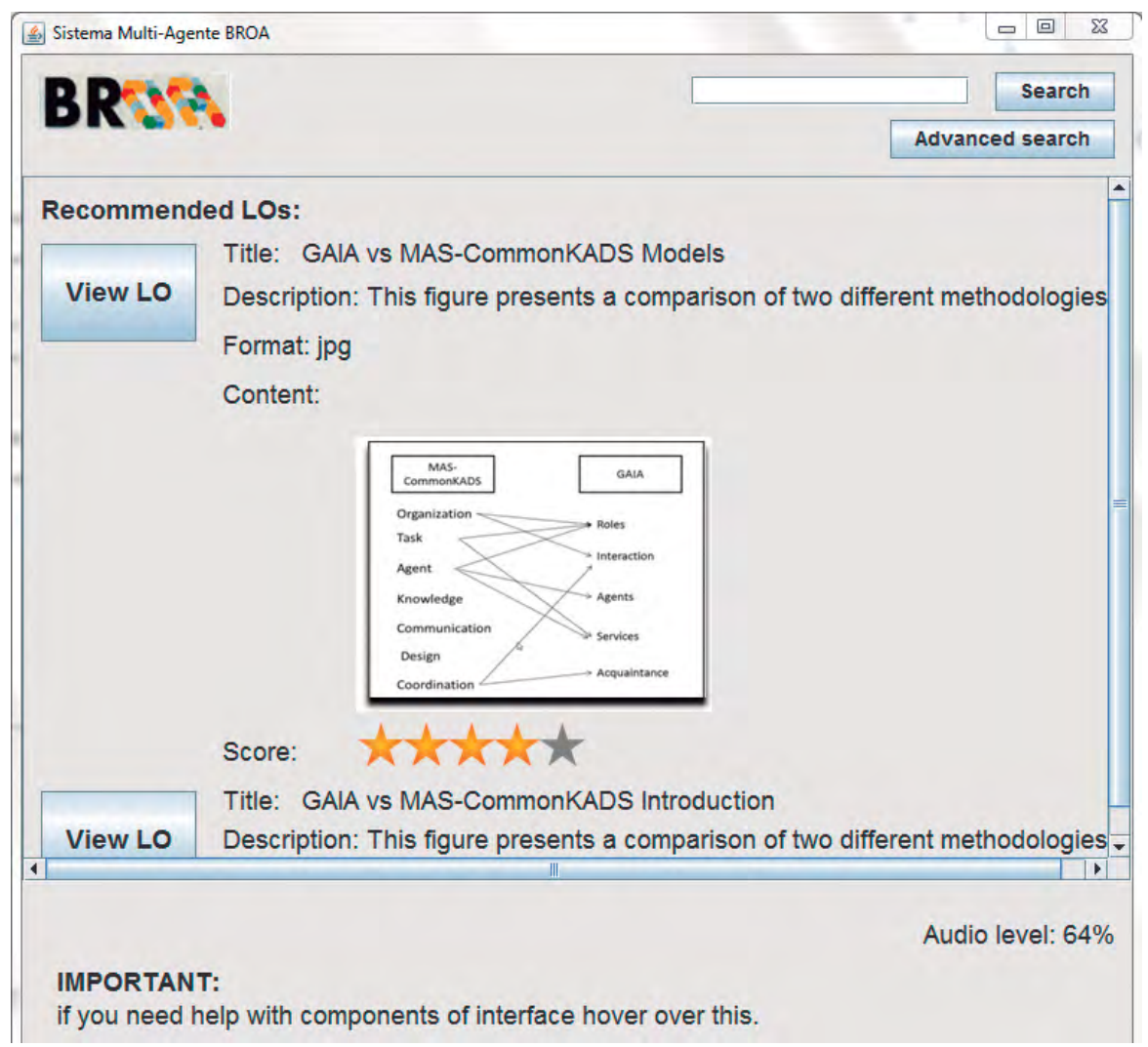

Figura 5. Interfaz adaptada al perfil del estudiante

Fuente: elaboración propia

\section{CONCLUSIONES}

A partir de la evaluación realizada a la interfaz adaptativa se puede concluir que: (a) la interfaz personaliza correctamente aspectos como color y tamaño del texto, idioma, nivel de volumen auditivo y distribución del contenido; (b) las características recopiladas en el perfil del estudiante permiten describir satisfactoriamente los gustos y necesidades, lo cual facilita la adaptación de la información contenida en la interfaz gráfica; (c) las recomendaciones obtenidas del sistema las cuales utilizan inferencias ontológicas son bastante adecuadas; (d) utilizar agentes móviles por cada repositorio facilita la recuperación y el procesamiento de los OA, así como de sus metadatos; (e) la personalización proactiva del contenido permite capturar la atención de los estudiantes, mejorando el proceso de aprendizaje dentro del entorno virtual.

Como trabajo futuro se propone ampliar los comportamientos del agente interfaz para que realice adaptaciones a interfaces de dispositivos móviles, con el fin de brindar al sistema características de ubicuidad y movilidad. De igual manera, utilizar plataformas como Android puede ser de gran utilidad al momento de adaptar tanto las interfaces como los OA, puesto que estas tecnologías ofrecen información contextual de gran utilidad como posición geoespacial, acelerómetro, cámara fotográfica, redes sociales, etc. 


\section{FINANCIAMIENTO}

El trabajo de investigación que se presenta en este artículo ha sido financiado parcialmente por el siguiente proyecto de COLCIENCIAS: "RAIM: Implementación de un framework apoyado en tecnologías móviles y de realidad aumentada para entornos educativos ubicuos, adaptativos, accesibles e interactivos para todos", registrado en la Universidad Nacional de Colombia, bajo el código 1119-569-34172.

\section{REFERENCIAS}

Becerra, C., Jiménez Vargas, S., González, F. y Gelbukh, A. (2015). Recomendación de productos a partir de perfiles de usuario interpretables. Revista Tecnura, 19(45), 89-100. doi: 10.14483/udistrital.jour. tecnura.2015.3.a07

Bellifemine, F., Poggi, A. and Rimassa, G. (1999). JADE-A FIPA-Compliant Agent Framework. Proceedings of PAAM.

Berners-Lee, T. and Hendler, J. (2001). Publishing on the Semantic Web. Nature, 410, pp. 1023-1024.

Bolotnikova, E., y Gavrilova, A. and Gorovoy, V. (2011). To a Method of Evaluating Ontologies. Journal of Computer and Systems Sciences International, 448-461.

IEEE. (2002). IEEE Standard for Learning Object Metadata. New York: Institute of Electrical and Electronics Engieneers.

Kolas, L. and Staupe, A. (2007). A Personalized E-learning Interface. Eurocon 2007-The International Conference on Computer as a Tool (pp. 26702675). IEEE.

Kritikou, Y., Demestichas, P., Adamopoulou, E., Demestichas, K., Theologou, M., and Paradia, M. (2008). User Profile Modeling in the Context of Web-Based Learning Management Systems. Journal of Network and Computer Applications, Volume 31, Issue 4, pp. 603-627.

Lee, H., Choi, Y. and Kim, Y. (2011). An Adaptive User Interface Based on Spatiotemporal Structure
Learning. Communications Magazine, IEEE, Vol. 49 (6):118-124.

Liang, Y., Zhu, H., Tian, Q., and Ji, S. (2010). A Method for OWL Ontology Module Partition. Web Society (SWS), 2010 IEEE 2nd Symposium on (pp. 372377). Beijing: IEEE.

Nivethika, M., Vithiya, I., Anntharshika, S., and Deegalla, S. (2013). Personalized and Adaptive User Interface Framework for Mobile Application. International Conference on Advances in Computing, Communications and Informatics (ICACCI) (pp. 1913-1918). IEEE.

Papanikolaou, K., Grigoriadou, M., Kornilakis, H., and Magoulas, G. (2002). Personalizing The Interaction in a Web-Based Educational Hypermedia System: The Case of INSPIRE Department of Information Systems and Computing. Information systems, Volume 13, Issue 3, pp. 213-267.

Peissner, M., and Edlin-White, R. (2013). User Control in Adaptive User Interfaces for Accessibility. Human-Computer Interaction - INTERACT 2013 (pp. 623-640). Springer.

Rodríguez, P., Tabares, V., Duque, N., Ovalle, D., and Vicari, R. (2013). BROA: An Agent-Based Model to Recommend Relevant Learning Objects from Repository Federations Adapted to Learner Profile. International Journal of Interactive Multimedia and Artificial Intelligence, Vol. 2, № 1, pp. 6-11.

Rosado, A., and Verjel, A. (2015). Data Mining Applied to Demand of Air Transport in Ocaña, North Santander. Revista Tecnura, 19(45), 101-113, http://dx.doi. org/10.14483/udistrital.jour.tecnura.2015.3.a08.

Smith, B., McGinty, L., Reilly, J., and McCarthy, K. (2004). Compound Critiques for Conversational Recommender Systems. Proceedings of the 2004 IEEE/WIC/ACM International Conference on Web Intelligence (pp. 145-151). Washington.

Steichen, B., Ashman, H., and Wade, V. (2012). A comparative survey of Personalised Information Retrieval and Adaptive Hypermedia techniques. Information Processing y Management, Vol. 48 (4), 698-724.

Uruchrutu, E., MacKinnon, L., and Rist, R. (2005). User Cognitive Style and Interface Design for Personal, 
Adaptive Learning. What to Model? 10th International Conference, UM 2005. Edinburgh: Proceedings. Van de Sompel, H., Chute, R., and Hochstenbach, P. (2008). The aDORe Federation Architecture: Digital Repositories at Scale. International Journal on Digital Libraries, 83-100.
Wooldridge, M., Jennings, N., and Kinny, D. (1999). A Methodology for Agent-Oriented Analysis and Design. Proceedings of the Third Annual Conference on Autonomous Agents (pp. 69-76). New York. 